\title{
Inhibition of hepatic stellate cells by bone marrow- derived mesenchymal stem cells in hepatic fibrosis
}

\author{
Yoon Ok Jang ${ }^{1,2^{*}}$, Baek Gyu Jun ${ }^{3^{*}}$, Soon Koo Baik ${ }^{1,2}$, Moon Young Kim', and Sang Ok Kwon \\ 'Department of Internal Medicine, Yonsei University Wonju College of Medicine, Wonju; ${ }^{2}$ Cell Therapy and Tissue Engineering Center, \\ Yonsei University Wonju College of Medicine, Wonju; ${ }^{3}$ Department of Internal Medicine, Cheonan Hospital, Soonchunhyang University \\ College of Medicine, Cheonan, Korea
}

Background/Aims: Therapies involving bone-marrow-derived mesenchymal stem cells (BM-MSCs) have considerable potential in the management of hepatic disease. BM-MSCs have been investigated in regenerative medicine due to their ability to secrete various growth factors and cytokines that regress hepatic fibrosis and enhance hepatocyte functionality. The aim of this study was to determine the antifibrosis effect of BM-MSCs on activated hepatic stellate cells (HSCs) and the mechanism underlying how BM-MSCs modulate the function of activated HSCs.

Methods: We used HSCs in both direct and indirect co-culture systems with BM-MSCs to evaluate the antifibrosis effect of BM-MSCs. The cell viability and apoptosis were evaluated by a direct co-culture system of activated HSCs with BM-MSCs. The activations of both HSCs alone and HSCs with BM-MSCs in the direct co-culture system were observed by immunocytochemistry for alpha-smooth muscle actin (a-SMA). The levels of growth factors and cytokines were evaluated by an indirect co-culture system of activated HSCs with BM-MSCs.

Results: The BM-MSCs in the direct co-culture system significantly decreased the production of a-SMA and the viability of activated HSCs, whereas they induced the apoptosis of activated HSCs. The BM-MSCs in the indirect co-culture system decreased the production of transforming growth factor- $\beta 1$ and interleukin (IL)- 6 , whereas they increased the production of hepatocyte growth factor and IL-10. These results confirmed that the juxtacrine and paracrine effects of BM-MSCs can inhibit the proliferative, fibrogenic function of activated HSCs and have the potential to reverse the fibrotic process by inhibiting the production of a-SMA and inducing the apoptosis of HSCS.

Conclusions: These results have demonstrated that BM-MSCs may exert an antifibrosis effect by modulating the function of activated HSCs. (Clin Mol Hepatol 2015;21:141-149)

Keywords: Bone-marrow-derived mesenchymal stem cell; Cirrhosis; Hepatic fibrosis

\section{INTRODUCTION}

Hepatic fibrosis, the precursor to cirrhosis, is the excessive deposition of extracellular matrix (ECM) and degradation mediated primarily by activated hepatic stellate cells (HSCs). ${ }^{1-4}$ Activated HSCs are generated in the ECM of the principal cells during the process of hepatic fibrosis.

In normal liver, HSCS are non-parenchymal, quiescent cells that

\section{Abbreviations: \\ $\mathrm{BM}$, bone marrow; MSCs, mesenchymal stem cells; BM-MSCs, bone marrow-derived mesenchymal stem cells; TGF- $\beta 1$, transforming growth factor-beta 1; a-SMA, alpha-smooth muscle actin; IL, interleukin; HGF, hepatocyte growth factor; SD, standard deviation}

\author{
Corresponding author : Soon Koo Baik \\ Department of Internal Medicine, Yonsei University, Wonju College of \\ Medicine, 20 Ilsan-ro, Wonju 220-701, Korea \\ Tel: +82-33-741-1223, Fax: +82-33-745-6782 \\ E-mail: baiksk@yonsei.ac.kr \\ *These authors contributed equally to this work.
}


have physiological functions as vitamin A storage, production of ECM in the space of Disse and a role in the regulation of the sinusoidal microcirculatory flow. ${ }^{5}$ However, during liver fibrogenesis, HSCs activate or transdifferentiate into myofibroblast-like cells, acquiring contractile and fibrogenic properties, secreting cytokines and expressing alpha-smooth muscle actin ( $\alpha$-SMA). ${ }^{6,7}$

The transforming growth factor- $\beta 1$ (TGF- $\beta 1$ ) is recognized as one of the main profibrogenic mediators ${ }^{8,9}$ and plays a key role in the HSC activation and subsequent hepatic fibrosis. As activated HSCs are the principal cells to produce collagen in fibrotic liver, they contribute to the development of hepatic fibrosis through juxtacrine and paracrine signals of TGF- $\beta$ stimulated collagen production.

Cell therapies have shown promising benefits for hepatic fibrosis in experimental and clinical studies. ${ }^{10-15}$ Recently, stem cell treatment has been suggested as an effective therapy for hepatic disease and mesenchymal stem cells (MSCs) are excellent candidates for cell therapy. Among the stem cells, MSCs in particular have practical advantages in regenerative medicine due to their high capability for self-renewal, their potential for multipotent differentiation, and their low immunogenicity.

Bone marrow-derived mesenchymal stem cells (BM-MSCs) are known to naturally support hematopoiesis by secreting a number of trophic molecules, including soluble extracellular matrix glycoproteins, cytokines, and growth factors. ${ }^{16,17}$ Previous studies have demonstrated that BM-MSCs therapy can ameliorates hepatic fibrosis and reverses fulminant hepatic failure and can effectively alleviate end-stage liver disease and improve symptoms and liver function, indicating the effectiveness and safety of BM-MSCs in clinical implantation. ${ }^{12,18,19}$

For therapeutic applications, it will be important to understand the potency and possible repair mechanisms of BM-MSCs to help us understand the nature of hepatic fibrosis. However, apart from the paracrine mechanism by which the anti-fibrosis effect of BMMSCs inhibit activated HSCs, the effects of juxtacrine and paracrine signaling between the two cell types are not clearly understood. The purpose of this study was to explore how the BMMSCs could modulate activated HSCs via in affecting hepatic fibrosis via direct and indirect co-culture system. In this study, we demonstrate that the anti-fibrosis effect of BM-MSCs on activated HSCs and the underlying mechanism by which BM-MSCs modulate the function of activated HSCs.

\section{MATERIALS AND METHODS}

\section{Preparation of human bone marrow-derived mesenchymal stem cells}

Human BM-MSCs were obtained from healthy persons who voluntarily donated their bone marrow stem cells. Approximately 10 $20 \mathrm{~mL}$ of BM was aspirated from the posterior iliac crests of humans under local anesthesia. BM mononuclear cells were isolated through density-gradient centrifugation (Histopaque-1077, SigmaAldrich, St. Louis, MO, USA). Mononuclear cells $\left(2-3 \times 10^{5}\right.$ cells/ $\mathrm{cm}^{2}$ ) were plated in a $75-\mathrm{cm}^{2}$ flask (Falcon, Franklin Lakes, NJ, USA) with Dulbecco's modified Eagle's medium (DMEM; Gibco, Grand Island, NY, USA) containing 10\% fetal bovine serum (FBS; Gibco) and 1\% penicillin/streptomycin (Gibco) and then cultured at $37^{\circ} \mathrm{C}$ in a $5 \% \mathrm{CO}_{2}$ atmosphere. When the cultures approached $80 \%$ confluence, the cells were harvested by treatment with a trypsin/EDTA solution (Gibco) and replated at a density of $4-5 \times 10^{3}$ cells $/ \mathrm{cm}^{2}$ in $175-\mathrm{cm}^{2}$ flasks. The human procedures and protocols were approved by the Institutional Review Board (IRB) at Yonsei University Wonju Severance Hospital (CR109021), and conducted according to the principles of the Declaration of Helsinki. All participants provided written informed consent prior to participation in the study.

\section{Immunophenotypes and differentiation assays of BM-MSCs}

The immunophenotypes of the BM-MSCs (CD14, CD34, CD45, CD73, and CD105) were analyzed prior to experimental use, and their differentiation potentials were determined (osteogenesis and adipogenesis; Fig. 1). For the immunophenotype analysis, BM-MSCs were stained with antibodies conjugated with fluorescein isothiocyanate (FITC) or phycoerythrin (PE): CD14-FITC, CD34-FITC, CD45-FITC, CD73-PE, and CD105-PE (BD Biosciences, San Jose, CA, USA). Briefly, $5 \times 10^{5}$ cells were resuspended in 0.2 $\mathrm{mL}$ of phosphate-buffered saline (PBS) and incubated with FITCor PE-conjugated antibodies for $20 \mathrm{~min}$ at room temperature. FITC- or PE-conjugated mouse lgGs were used as the control isotype at the same concentration as the specific primary antibodies. The fluorescence intensity of the cells was evaluated through flow cytometry (Epics XL; Beckman Coulter, Miami, FL, USA). Osteogenic differentiation was determined by first plating the cells at $2 \times 10^{4}$ cells $/ \mathrm{cm}^{2}$ in six-well plates and then incubating them in the following osteogenic medium for 2-3 weeks: DMEM (Gibco, 

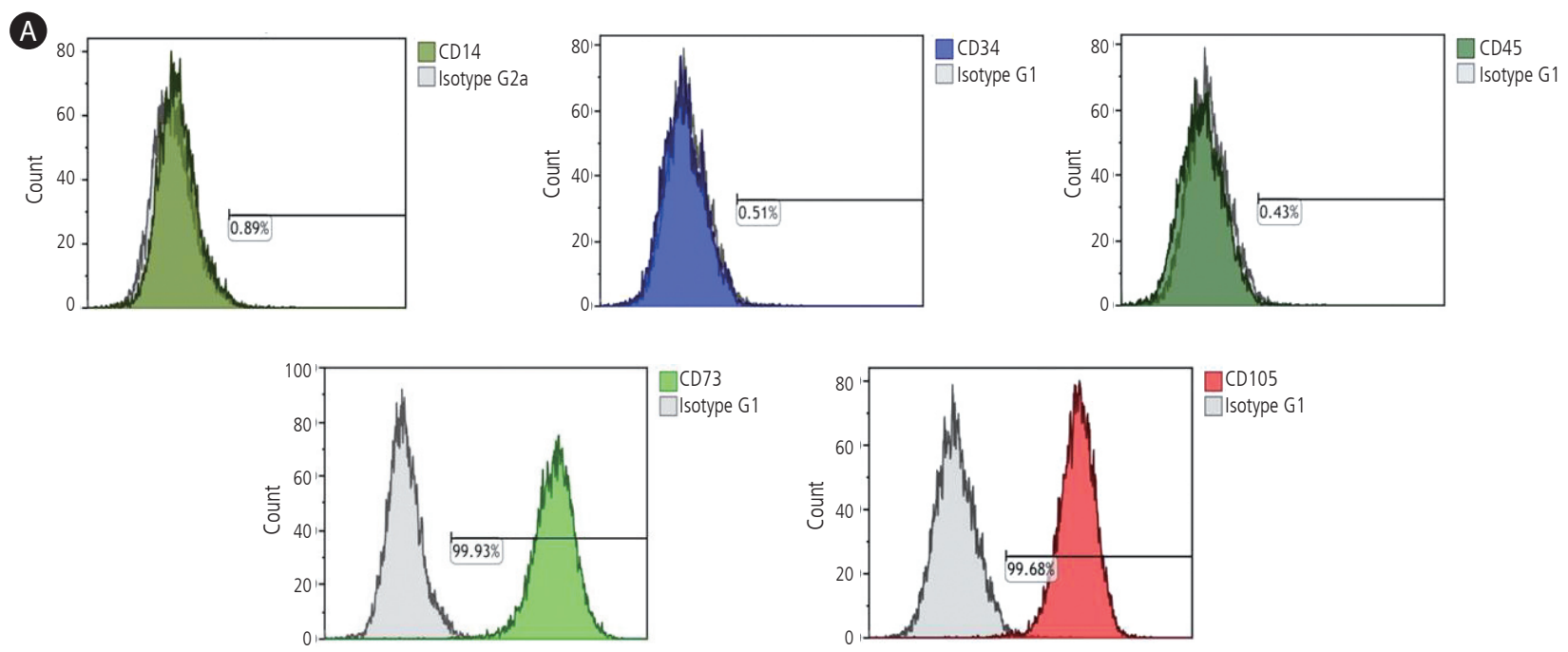

$\square$ Isotype G1

Fluorescence intensity

B

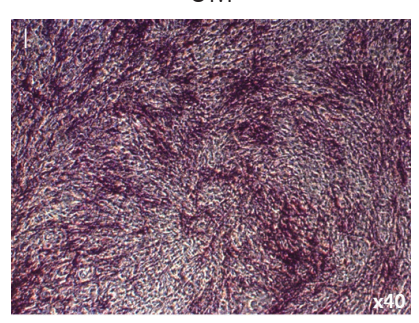

CM

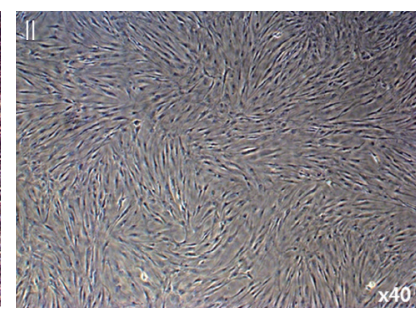

AM

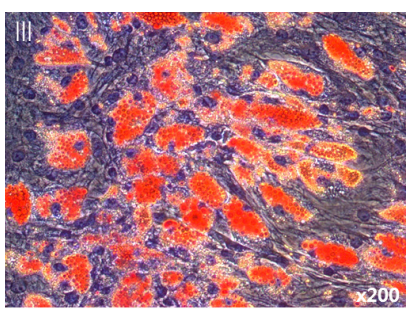

$\mathrm{CM}$

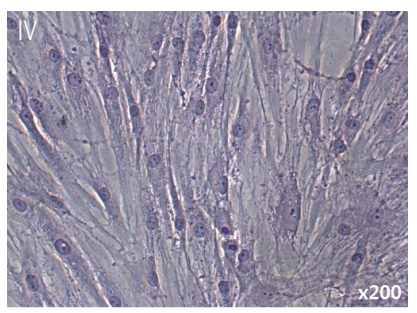

Figure 1. Immunophenotypes and differentiation potentials of BM-MSCs. (A) The expressions of cell-surface antigens (CD14, CD34, CD45, CD73, and CD105) were evaluated by flow cytometry. (B) BM-MSCs stained positively for endogenous alkaline phosphatase activity, indicating osteogenic differentiation within an osteogenic medium (OM; I), or stained negatively in control medium (CM; II) (X40). BM-MSCs stained positively for lipid droplets, indicating adipogenic differentiation within adipogenic medium (AM; III), or stained negatively in CM (IV) (×200).

Grand Island, NY, USA) supplemented with 10\% FBS, $10 \mathrm{mM}$ $\beta$-glycerophosphate, $10^{-7} \mathrm{M}$ dexamethasone, and $0.2 \mathrm{mM}$ ascorbic acid (Sigma-Aldrich). ${ }^{20}$ The osteogenic differentiation was quantified from the release of $p$-nitrophenol from $p$-nitrophenyl phosphate by the enzyme alkaline phosphatase. ${ }^{21}$ For adipogenic differentiation, BM-MSCs were plated at $2 \times 10^{4} \mathrm{cells} / \mathrm{cm}^{2}$ in sixwell plates and cultured for 1 week; then, differentiation was induced with adipogenic medium $110 \%$ FBS, $1 \mu \mathrm{M}$ dexamethasone, $0.5 \mathrm{mM}$ 3-isobutyl-1-methylxanthine, $10 \mu \mathrm{g} / \mathrm{mL}$ insulin, and $100 \mu \mathrm{M}$ indomethacin in high-glucose DMEM) for an additional 3 weeks. The differentiated cells were fixed in $4 \%$ paraformaldehyde for 10 min and stained with fresh Oil Red-0 solution (Sigma-Aldrich) to display lipid droplets (Fig. 1B). The criteria regarding the use of MSCs included viability greater than $80 \%$; the absence of microbial contamination (bacteria, fungus, virus, or mycoplasma) when tested 3-4 days before experimentation; CD73 and CD105 expression in more than 90\% of the cells; and the absence of CD14, CD34, and CD45 in less than 3\% of the cells, as assessed by flow cytometry.

\section{HSCs culture}

We used immortalized human HSCs that were established by retroviral expression of human telomerase reverse transcriptase (hTERT) for the majority of in vitro experiments. Characterization by immunofluorescence for $\alpha$-SMA revealed a purity of $>96 \%$. HSCs were plated in a $75 \mathrm{~cm}^{2}$ flask (Falcon) with DMEM (Gibco) containing 10\% FBS (Gibco) and 1\% penicillin/streptomycin (Gibco), and cultured at $37^{\circ} \mathrm{C}$ in a $5 \% \mathrm{CO}_{2}$ atmosphere.

\section{Co-culture system}

For co-culture system of HSCs and BM-MSCs, cells were seeded at a 1:1 ratio in each well of a 12 or 96 well plate (Costar, Corning 
Inc., Corning, NY, USA). An indirect co-culture system of HSCs and BM-MSCs was assembled using Transwell membranes (12 mm diameter, $0.4 \mu \mathrm{m}$ pore size, Costar, Corning Inc., Corning, NY, USA). HSCs were placed in the lower chamber with BM-MSCs placed on the membrane insert. Indirect co-culture system were maintained in DMEM for 48 hrs.

\section{Cytokine levels analysis}

An indirect co-culture system of activated HSCs with BM-MSCs were maintained in DMEM containing 10\% FBS at a density of 1:1 for 48 hrs. Supernatants were collected after 48 hrs of indirect co-culture system. Quantification of human TGF- $\beta 1$, interleukin (IL)-6, IL-10, and hepatocyte growth factor (HGF) from culture supernatant was determined using enzyme-linked immunosorbent assay kit (R\&D Systems, Minneapolis, MN, USA), following the manufacturer's instructions. The absorbance of each well was measured at $490 \mathrm{~nm}$ wavelength using a microplate reader ELX 800 (Bio-Tek Instruments, Inc., Winooski, VT, USA).

\section{Viability analysis}

Cell viability assays performed using a CellTiter $96^{\circledR}$ AQueous One Solution Reagent ELISA kit (Promega, Madison, WI, USA). The CellTiter 96 Aqueous One Solution contains MTS [3-(4,5-dimethylthiazol-2-yl)-5-(3-carboxymethoxyphenyl)-2-(4-sulfophenyl)-2H-tetrazolium, inner salt] compound and an electron coupling reagent phenazine ethosulfate (PES). The MTS compound is bioreduced by mitochondrial enzymes of cells into a colored formazan product that is soluble in cell culture medium. Briefly, for direct co-culture system of HSCs and BM-MSCs, cells were seeded at a 1:1 ratio in each well of a 96 well plate (Corning). Co-cultures were maintained in DMEM for 4 days. Assays are performed by adding a small amount of the CellTiter $96^{\circledR}$ AQueous One Solution Reagent direct to culture wells, incubating for $2 \mathrm{hrs}$ and then recording the absorbance at $490 \mathrm{~nm}$ wavelength using a microplate reader ELX 800 (Bio-Tek Instruments).

\section{Apoptosis analysis}

Quantification of cell apoptosis was measured using a Cell Death Detection ELISA kit (Roche Applied Science, Indianapolis, IN, USA). Cell Death Detection ELISA kit was used to quantify histone-complexed DNA fragments (nucleosomes) in cytoplasm of the apoptotic cells after induction of apoptosis. Briefly, for di- rect co-culture system of HSCs and BM-MSCs, cells were seeded at a 1:1 ratio in each well of a 12 well plate (Corning). After direct co-culture system of HSCs and BM-MSCs for 4 days, cells were lysed with cell lysis buffer. Monoclonal antibodies against singlestrand DNA and histones ( $\mathrm{H} 1, \mathrm{H} 2 \mathrm{a}, \mathrm{H} 2 \mathrm{~b}, \mathrm{H} 3$, and $\mathrm{H} 4)$ specifically detected and bound mononucleosomes and oligonucleosomes derived from cells undergoing apoptosis. Biotinylated antihistone antibodies then fixed the antibody-nucleosome complexes to the streptavidin-coated microtiter plate. The anti-DNA antibodies were conjugated with a peroxidase that reacted with the substrate ABTS (2,2V-azino-di[3-ethylbenzthiazolinsulfonate]) to form a colored product. The remaining steps were carried out according to the instructions supplied by the manufacturer. The absorbance of each well was measured at $405 \mathrm{~nm}$ wavelength using a microplate reader ELX 800 (Bio-Tek Instruments).

\section{Immunocytochemistry}

Cells were cultured on 4-well chamber slide (BD Falcon, Bedford, MA, USA) and treated with or without TGF- $\beta 1$ (R\&D Systems, Abingdon, UK). After $24 \mathrm{hrs}$ of TGF- $\beta 1$ (1 ng/mL) treatment, cells were washed three times with PBS and fixed with $4 \%$ paraformaldehyde in PBS for 15 min and followed by incubation with Protein Block for 15 min at room temperature. The cells were incubated with anti- $\alpha$-SMA monoclonal antibody (Abcam, Cambridge, MA, USA) overnight at $4^{\circ} \mathrm{C}$. The cells were washed with PBS and incubated with Alexa Fluor ${ }^{\circledR} 488$ (Invitrogen, Carlsbad, (A, USA) secondary antibody for $1 \mathrm{hr}$ at room temperature in dark room. After washing, the slides were mounted with Vectashield (Vector Labs, Burlingame, CA, USA) mounting media containing 4',6-diamino-2-phenylindole (DAPI) for detection of all nuclei. Fluorescence images were observed with a laser scanning confocal microscope (TCS SPE, Leica Microsystems GmbH, Wetzlar, Germany).

\section{Statistical analysis}

Values are expressed as means \pm standard deviation. Analysis was conducted using nonparametric analysis with the MannWhitney $U$ tests. All analyses were performed using SPSS software version 18.0 (SPSS Inc., Chicago, IL, USA). For all tests, $P$ values of $<0.01$ were considered statistically significant. 
Yoon Ok Jang, et al. Bone marrow-derived mesenchymal stem cells for hepatic fibrosis

\section{a-SMA}
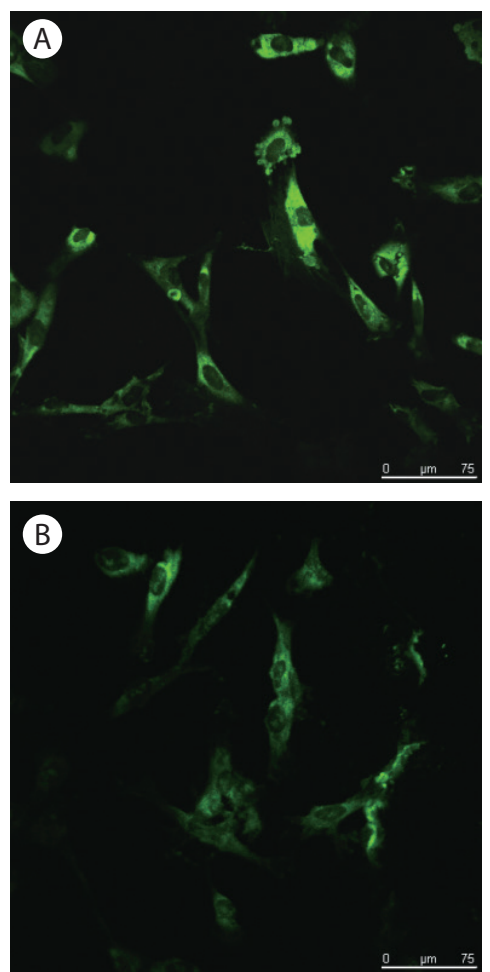

(C)

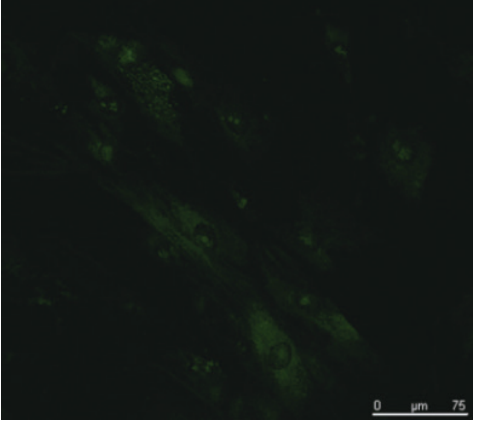

(D)

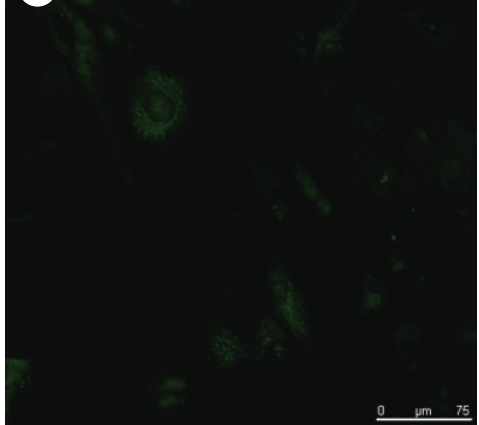

DAPI
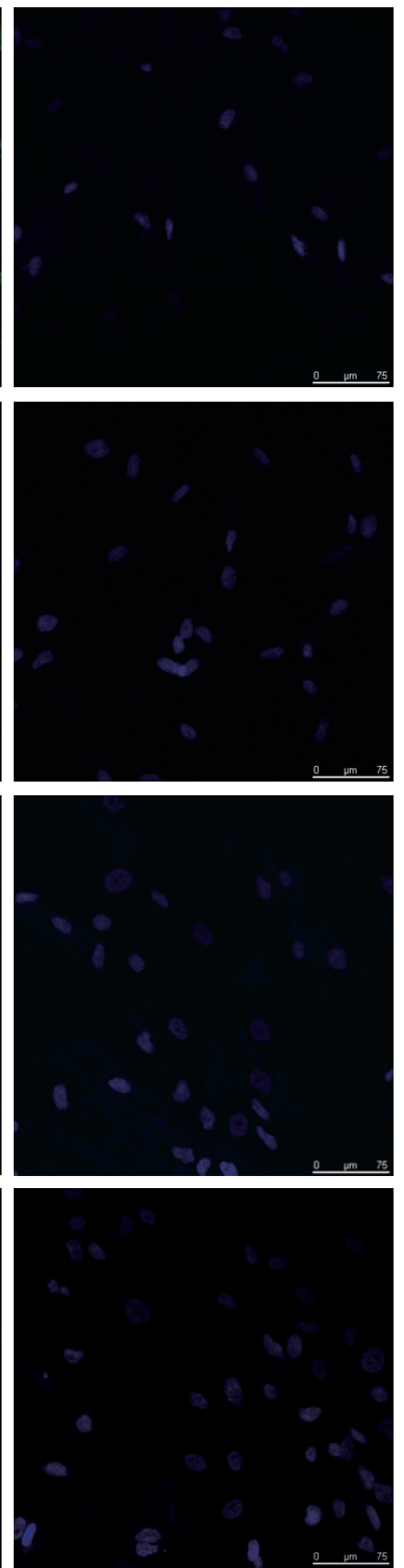

Merge
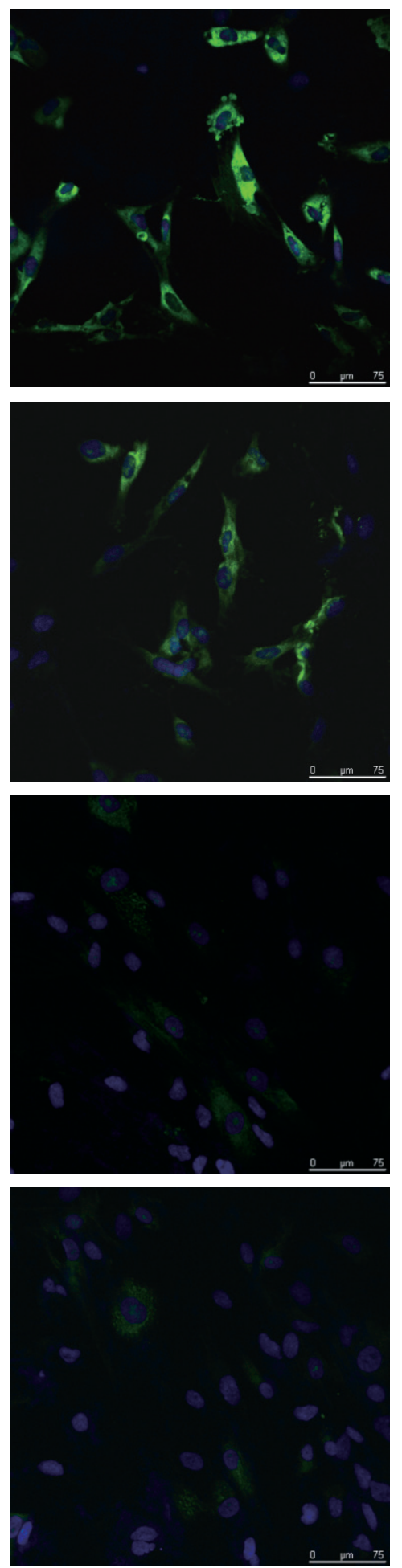

Figure 2 Expression of a-SMA in the direct co-culture system of activated HSCs with BM-MSCs as measured by immunocytochemistry. The expression of a-SMA showed that (A, B) activated HSCs and (C, D) activated HSCs with BM-MSCs by fluorescent immunocytochemistry. The expression of a-SMA in cells treated $(A, C)$ with or $(B, D)$ without TGF- $\beta 1$. Nuclei were stained with DAPI. Merged immunofluorescence images of a-SMA (green) and DAPI (blue). (Scale bar, $75 \mu \mathrm{m}$ ). a-SMA, alpha-smooth muscle actin; HSCs, hepatic stellate cells; BMMSCs, bone marrow-derived mesenchymal stem cells; TGF- $\beta 1$, transforming growth factor-beta 1; DAPI, 6-diamino-2-phenylindole. 
A

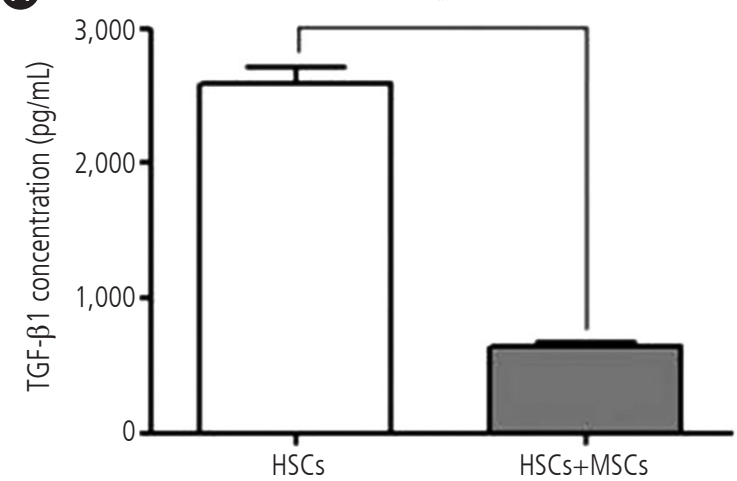

C

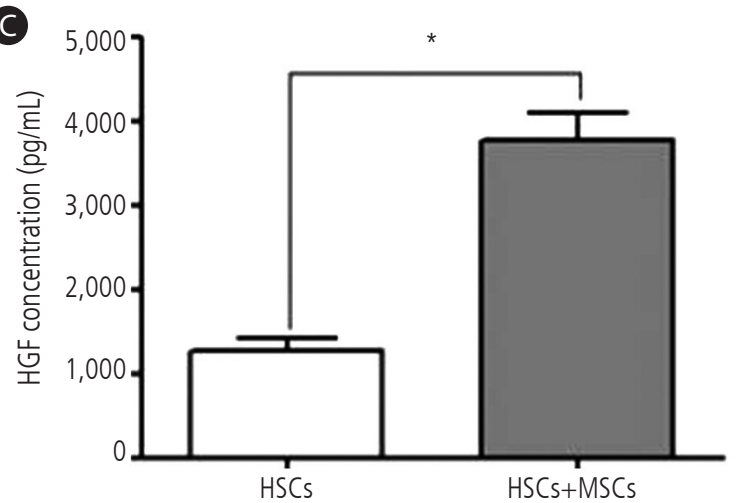

B

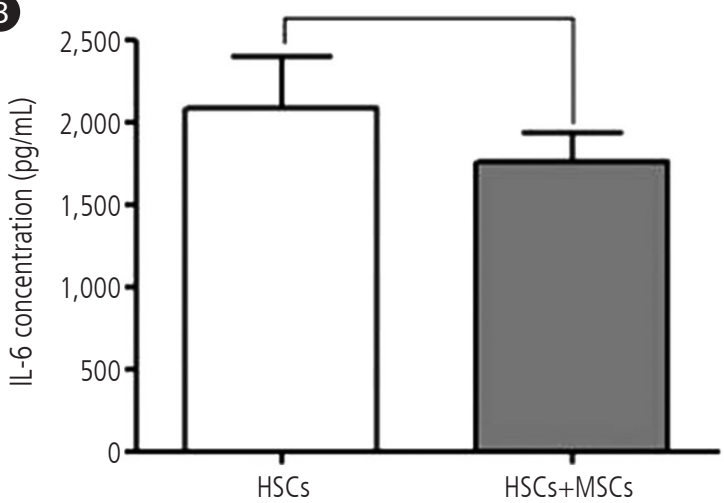

(D)

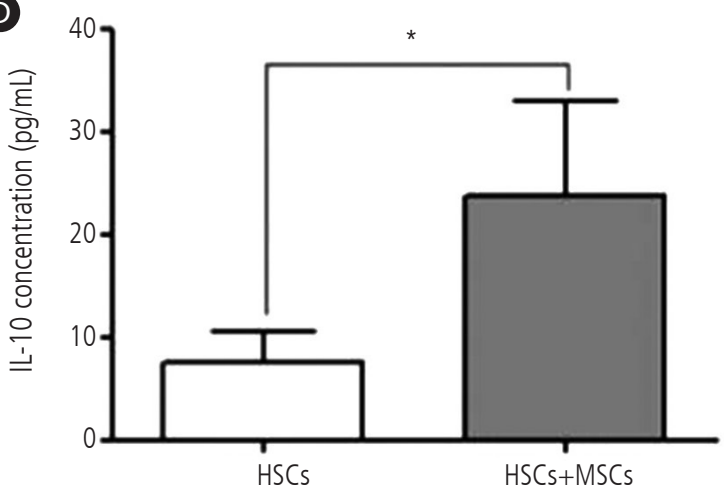

Figure 3. Cytokine levels of TGF- $\beta 1$, HGF, IL-6, and IL-10. Indirect co-culture system of activated HSCs with BM-MSCs decreased the production of (A) TGF- $\beta 1$ and (B) IL-6. Whereas, co-culture system of activated HSCs with BM-MSCs increased the production of (C) HGF and (D) IL-10. Values are presented as mean $\pm \mathrm{SD}$. ${ }^{P}<0.01$. HSCs, hepatic stellate cells; MSCs, mesenchymal stem cells; BM-MSCs, bone marrow-derived mesenchymal stem cells.

\section{RESULTS}

\section{Immunophenotypes and differentiation potentials of the BM-MSCs}

The immunophenotypes for CD14, CD34, CD45, CD73, and CD105 cells were determined and osteogenic or adipogenic differentiation was induced on experimental the day (Fig. 1). In cell populations, CD73 or CD105 (which are positive markers of BMMSCs) were expressed in more than $99 \%$ of the cells. However, CD14, CD34, or CD45 (which are known to be negative markers of BM-MSCS) were expressed in less than $1 \%$ of the cells (Fig. 1A). BM-MSCs could be differentiated into osteocytes and adipocytes (Fig. 1B).

\section{Inhibition of the activation of HSCs by BM-MSCs}

The expression of $\alpha$-SMA, a specific marker for activated HSCs was observed by fluorescent immunocytochemistry. We observed that the activation of HSCS resulted in the expression of $\alpha$-SMA, which more increased by TGF- $\beta 1$ treatment (Fig. 2A and 2B). The expression of $\alpha$-SMA in direct co-culture system of activated HSCs with BM-MSCs were significantly decreased compared to the HSCs (Fig. 2).

\section{The cytokine levels of TGF- $\beta 1$, HGF, IL-6, and IL-10}

To determine whether BM-MSC paracrine factors could modulate activated HSCs via indirect co-culture system. Measurement of TGF- $\beta 1$, HGF, IL- 6 , and IL-10 from supernatant of co-cultured medium were done by ELISA. An indirect co-culture system of activated HSCs with BM-MSCs decreased the production of TGF- $\beta 1$ by $75 \%$ and IL- 6 by $16 \%$, respectively $(P<0.01$, Fig. 3$)$. Whereas, co-culture system of activated HSCs with BM-MSCs increased the production of HGF by 3.0-fold and IL-10 by 3.2-fold, respectively $(P<0.01$, Fig. 3). 
A

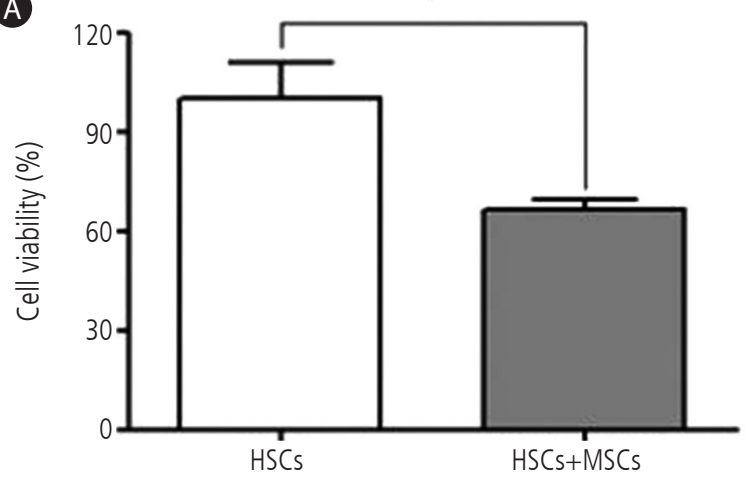

B

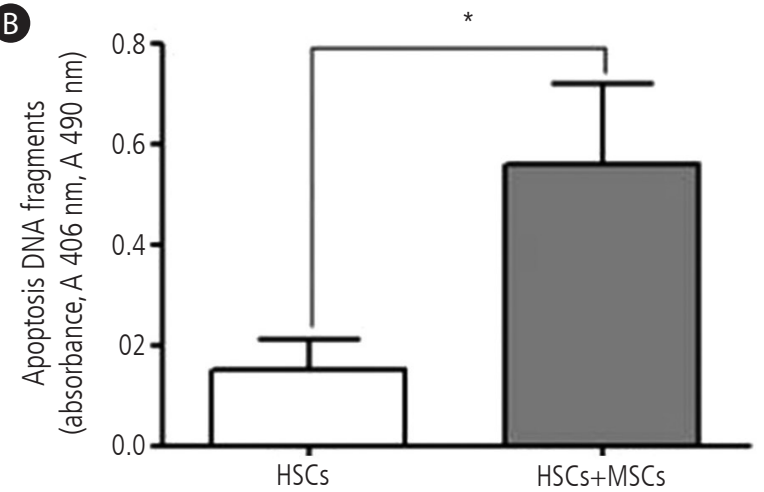

Figure 4. Inhibition of viability and induction of apoptosis in activated HSCs by BM-MSCs. (A) The HSCs viability was decreased and (B) apoptosis was increased with direct co-culture system of activated HSCs with BM-MSCs. Values are presented as mean $\pm \mathrm{SD}$. ${ }^{*} P<0.01$. HSCs, hepatic stellate cells; MSCs, mesenchymal stem cells; BM-MSCs, bone marrow-derived mesenchymal stem cells.

\section{Inhibition of viability and induction apoptosis in activated HSCs by BM-MSCs}

At a 1:1 co-culture ratio, to determine whether BM-MSCs have the capacity to inhibit activated HSCs viability, we quantified the CellTiter $96^{\circledR}$ AQueous One Solution Reagent ELISA kit. And to determine whether BM-MSCs also have the capacity to reduce activated HSCs numbers by inducing their apoptosis, we quantified the Cell Death Detection ELISA kit. HSCs viability was decreased by $34 \%$ and apoptosis was increased by 3.7-fold with direct co-culture system of activated HSCs with BM-MSCs, respectively $(P<0.01)$. We confirmed that BM-MSCs induced apoptosis of HSCs and also significantly inhibited viability of HSCs (Fig. 4).

\section{DISCUSSION}

BM-MSCs can produce a series of growth factors, cytokines and signal molecules that can potentially suppress inflammatory responses, reduce hepatocyte apoptosis, regress hepatic fibrosis and stimulate the proliferation of endogenous hepatocytes. ${ }^{22} \mathrm{BM}$ MSCs have the ability to prevent the progress of hepatic fibrosis, enhance hepatocyte functionality, and repair damaged liver.

$\alpha$-SMA is not only a marker for portal myofibroblasts, but also for activated HSCs which is recognized as a key player in hepatic fibrosis and cirrhosis. ${ }^{23}$ In this study showed that the expression of $\alpha$-SMA in direct co-culture system of activated HSCs with BMMSCS were significantly decreased compared to the activated HSCs (Fig. 2). Through immunofluorescence staining revealing $\alpha$-SMA expression, these results were confirmed that HSCs activa- tion was inhibited when these cells were co-cultured with BMMSCs. These results were demonstrated that BM-MSCs have the potential to reverse the fibrotic process by inhibiting $\alpha$-SMA production.

In vivo activation of HSCs is divided into a fibrogenic and hyperplastic response ${ }^{24}$ that is mediated by many autocrine and paracrine signals. HSCs not only secrete cytokines but also respond to them, emphasizing the importance of tightly regulated local control of cytokine action within the pericellular milieu. Previous studies have shown that BM-MSCs modulate the functions of the activity of HSCs with specific cytokines and growth factors such as TGF- $\beta 1, \mathrm{IL}-6, \mathrm{IL}-10$, and HGF. ${ }^{25}$

TGF- $\beta 1$ is a primary mediator particularly in liver fibrogenesis. ${ }^{26}$ HSCs secrete latent TGF- $\beta 1$ in response to injury, which, after its activation, exerts potent fibrogenic effects in both autocrine and paracrine patterns. In the liver, IL-6 induces hepatic acute phase protein synthesis in vivo and IL- 6 is an important proinflammatory cytokine that have been associated with hepatic fibrosis. ${ }^{27} \mathrm{IL}-10$ is protecting hepatocytes, inhibiting the synthesis of pro-inflammatory cytokines, promoting ECM degradation and modulating hepatic fibrogenesis. ${ }^{28,29}$ HGF was identified as a mitogen for mature hepatocytes, ${ }^{30}$ and is considered to be important for the development and regeneration of liver. ${ }^{31}$ HGF suppressed proliferation and stimulated apoptotic cell death in liver myofibroblasts. ${ }^{32}$

Consistent with their result, our data found that indirect co-culture system of activated HSCS with BM-MSCs decreased the production of TGF- $\beta 1$ and IL- 6 , whereas increased the production of HGF and IL-10. These results were correlated with prior study that BM-MSCs can exert a protective role through paracrine signaling to $\mathrm{HSCs}^{33}$ These data support that BM-MSCs have effect in reversing hepatic fibrosis with decreasing production of TGF- $\beta 1$, 
IL-6 and increasing production of HGF, IL-10. Furthermore, we can hypothesize that BM-MSCs may modulate the proliferation, viability and apoptosis of HSCs through paracrine signaling by cytokines and growth factors secreted by BM-MSCs. In present study, BM-MSCs induced apoptosis of HSCs and also significantly inhibited viability of HSCs in direct co-culture system. This data presented that BM-MSCs had strong inhibitory activities of HSCs and there were direct interactions between activated HSCs and BMMSCs through direct cell-to-cell contact.

In conclusion, these results support that the juxtacrine and paracrine effects of BM-MSCs can inhibit the proliferative, fibrogenic function of activated HSCs and have the potential to reverse the fibrotic process by inhibiting a-SMA production and induce HSCs apoptosis.

\section{Acknowledgements}

This study was supported by a grant of the Yonsei University Future-leading Research Initiative of 2014 and also by the Yonsei University Wonju College of Medicine Research Fund of 2014. The authors appreciate Prof. YH Paik (Samsung Medical Center, Sungkyunkwan University School of Medicine, Seoul, Korea) for providing the HSCs.

\section{Conflicts of Interest}

The authors have no conflicts to disclose.

\section{REFERENCES}

1. Schiff ER, Lee SS, Chao YC, Kew Yoon S, Bessone F, Wu SS, et al. Long-term treatment with entecavir induces reversal of advanced fibrosis or cirrhosis in patients with chronic hepatitis B. Clin Gastroenterol Hepatol 2011;9:274-276.

2. Chang TT, Liaw YF, Wu SS, Schiff E, Han KH, Lai CL, et al. Long-term entecavir therapy results in the reversal of fibrosis/cirrhosis and continued histological improvement in patients with chronic hepatitis B. Hepatology 2010;52:886-893.

3. Kim MY, Baik SK, Yea CJ, Lee IY, Kim HJ, Park KW, et al. Hepatic venous pressure gradient can predict the development of hepatocellular carcinoma and hyponatremia in decompensated alcoholic cirrhosis. Eur J Gastroenterol Hepatol 2009;21:1241-1246.

4. Kim MY, Suk KT, Baik SK, Kim HA, Kim YJ, Cha SH, et al. Hepatic vein arrival time as assessed by contrast-enhanced ultrasonography is useful for the assessment of portal hypertension in compensated cirrhosis. Hepatology 2012;56:1053-1062.
5. Geerts A. History, heterogeneity, developmental biology, and functions of quiescent hepatic stellate cells. Semin Liver Dis 2001;21:311335.

6. Bataller R, Brenner DA. Hepatic stellate cells as a target for the treatment of liver fibrosis. Semin Liver Dis 2001;21:437-451.

7. Knittel T, Kobold D, Piscaglia F, Saile B, Neubauer K, Mehde M, et al. Localization of liver myofibroblasts and hepatic stellate cells in normal and diseased rat livers: distinct roles of (myo-)fibroblast subpopulations in hepatic tissue repair. Histochemistry and cell biology 1999;112:387-401.

8. Bataller R, Brenner DA. Liver fibrosis. J Clin Invest 2005;115:209218.

9. El-Lakkany NM, Hammam OA, El-Maadawy WH, Badawy AA, AinShoka AA, Ebeid FA. Anti-inflammatory/anti-fibrotic effects of the hepatoprotective silymarin and the schistosomicide praziquantel against Schistosoma mansoni-induced liver fibrosis. Parasit Vectors 2012;5:9.

10. Kharaziha P, Hellstrom PM, Noorinayer B, Farzaneh F, Aghajani K, Jafari $F$, et al. Improvement of liver function in liver cirrhosis patients after autologous mesenchymal stem cell injection: a phase I-II clinical trial. Eur J Gastroenterol Hepatol 2009;21:1199-1205.

11. Pai M, Zacharoulis D, Milicevic MN, Helmy S, Jiao LR, Levicar $N$, et al. Autologous infusion of expanded mobilized adult bone marrowderived CD34+ cells into patients with alcoholic liver cirrhosis. Am J Gastroenterol 2008;103:1952-1958.

12. Terai S, Ishikawa T, Omori K, Aoyama K, Marumoto Y, Urata Y, et al. Improved liver function in patients with liver cirrhosis after autologous bone marrow cell infusion therapy. Stem cells 2006;24:22922298.

13. Cho KA, Lim GW, Joo SY, Woo SY, Seoh JY, Cho SJ, et al. Transplantation of bone marrow cells reduces $\mathrm{CCl} 4$-induced liver fibrosis in mice. Liver international : official journal of the International Association for the Study of the Liver 2011;31:932-939.

14. Jang YO, Kim YJ, Baik SK, Kim MY, Eom YW, Cho MY, et al. Histological improvement following administration of autologous bone marrow-derived mesenchymal stem cells for alcoholic cirrhosis: a pilot study. Liver Int 2014;34:33-41.

15. Jang Y, Kim M, Cho M, Baik S, Cho Y, Kwon S. Effect of bone marrow-derived mesenchymal stem cells on hepatic fibrosis in a thioacetamide-induced cirrhotic rat model. BMC Gastroenterol 2014;14:198.

16. Dorshkind K. Regulation of hemopoiesis by bone marrow stromal cells and their products. Annu Rev Immunol 1990:8:111-137.

17. Haynesworth SE, Baber MA, Caplan Al. Cytokine expression by human marrow-derived mesenchymal progenitor cells in vitro: effects of dexamethasone and IL-1 alpha. J Cell Physiol 1996;166:585-592.

18. Gaia S, Smedile A, Omede P, Olivero A, Sanavio F, Balzola F, et al. Feasibility and safety of G-CSF administration to induce bone 
marrow-derived cells mobilization in patients with end stage liver disease. J Hepatol 2006;45:13-19.

19. Gordon MY, Levicar N, Pai M, Bachellier P, Dimarakis I, Al-Allaf F, et al. Characterization and clinical application of human CD34+ stem/ progenitor cell populations mobilized into the blood by granulocyte colony-stimulating factor. Stem cells 2006;24:1822-1830.

20. Hu Y, Liao L, Wang Q, Ma L, Ma G, Jiang $X$, et al. Isolation and identification of mesenchymal stem cells from human fetal pancreas. J Lab Clin Med 2003;141:342-349.

21. Martin JY, Dean DD, Cochran DL, Simpson J, Boyan BD, Schwartz Z. Proliferation, differentiation, and protein synthesis of human osteoblast-like cells (MG63) cultured on previously used titanium surfaces. Clinical Oral Implants Res 1996;7:27-37.

22. Zhou P, Hohm S, Olusanya Y, Hess DA, Nolta J. Human progenitor cells with high aldehyde dehydrogenase activity efficiently engraft into damaged liver in a novel model. Hepatology 2009;49:1992-2000.

23. Friedman SL. Seminars in medicine of the Beth Israel Hospital, Boston. The cellular basis of hepatic fibrosis. Mechanisms and treatment strategies. N Engl J Med 1993;328:1828-1835.

24. Grotendorst GR, Rahmanie H, Duncan MR. Combinatorial signaling pathways determine fibroblast proliferation and myofibroblast differentiation. FASEB J 2004;18:469-479.

25. Lin H, Xu R, Zhang Z, Chen L, Shi M, Wang FS. Implications of the immunoregulatory functions of mesenchymal stem cells in the treatment of human liver diseases. Cell Mol Immunol 2011;8:19-22.
26. Wrana JL. Transforming growth factor-beta signaling and cirrhosis. Hepatology 1999;29:1909-1910.

27. Choi I, Kang HS, Yang Y, Wu CL, Cho CL, Lu CN, et al. IL-6 induces hepatic inflammation and collagen synthesis in vivo. Clin Exp Immunol 1994;95:530-535.

28. Hung KS, Lee TH, Chou WY, Wu CL, Cho CL, Lu CN, et al. Interleukin-10 gene therapy reverses thioacetamide-induced liver fibrosis in mice. Biochem Biophys Res Commun 2005;336:324-331.

29. Safadi R, Ohta M, Alvarez CE, Fiel MI, Bansal M, Mehal WZ, et al. Immune stimulation of hepatic fibrogenesis by CD8 cells and attenuation by transgenic interleukin-10 from hepatocytes. Gastroenterology 2004;127:870-882.

30. Nakamura T, Nishizawa T, Hagiya M, Seki T, Shimonishi M, Sugimura A, et al. Molecular cloning and expression of human hepatocyte growth factor. Nature 1989;342:440-443.

31. Michalopoulos GK, DeFrances MC. Liver regeneration. Science 1997;276:60-66.

32. Kim WH, Matsumoto K, Bessho K, Nakamura T. Growth inhibition and apoptosis in liver myofibroblasts promoted by hepatocyte growth factor leads to resolution from liver cirrhosis. Am J Pathol 2005;166:1017-1028.

33. Parekkadan B, van Poll D, Megeed Z, Kobayashi N, Tilles AW, Ber-

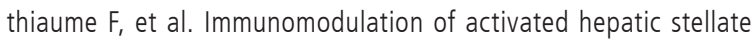
cells by mesenchymal stem cells. Biochem Biophys Res Commun 2007;363:247-252 